\title{
A State and Parameter Identification Scheme for Linearly Parameterized Systems
}

\author{
Chia-Shang Liu and Huei Peng \\ Department of Mechanical Engineering and Applied Mechanics \\ University of Michigan \\ hpeng@umich.edu
}

\begin{abstract}
This paper presents an adaptive algorithm to estimate states and unknown parameters simultaneously for nonlinear time invariant systems which depend affinely on the unknown parameters. The system output signals are filtered and re-parameterized into a regression form from which the least squares error scheme is applied to identify the unknown parameters. The states are then estimated by an observer based on the estimated parameters. The major difference between this algorithm and existing adaptive observer algorithms is that the proposed algorithm does not require any special canonical forms or rank conditions. However, an output measurement condition is imposed. The stability and performance limit of this scheme are analyzed. Two examples are then presented to show the effectiveness of the proposed schemes.
\end{abstract}

\section{Introduction}

In the monitoring and control of dynamic systems, frequently only a subset of the states are measured. To estimate the unknown states, state observers can be constructed, whose performance rely heavily on the accuracy of the plant model. For many practical problems, the system parameters are either unknown or time-varying. To achieve higher observer performance, it is necessary to estimate the states and unknown parameters simultaneously. Another motivation to estimate system parameters is that for many applications, knowing the values of certain parameters is important for monitoring and diagnosis purposes. The observers that simultaneously estimate states and parameters are frequently referred to as adaptive observers (Narendra and Annaswamy 1989) in the literature.

Adaptive observer algorithms have been widely studied since the early 1970's (e.g., Kudva and Narendra 1973, Luders and Narendra 1973, Kreisselmeier 1977, Dugard and Goodwin 1985, Marino and Tomei 1995). The Lyapunov approach, which provides guaranteed stability, has been a major tool for modern controller/observer designs. A key idea behind these adaptive observer methods is to construct a Luenberger observer (Luenberger 1971) whose parameters are updated in real-time. If the input is persistently exciting, the parameter estimation error may also converge to zero.

Depending on the availability of states, existing adaptive observer schemes can be classified into full-state feedback schemes and output feedback schemes. The focus of fullstate feedback schemes is on parameter identification and strictly speaking they do not qualify as state observers. The output feedback algorithms developed in the past are usually limited to special subgroups of systems. For example, the system may have to be strictly positive real (Narendra and Annaswamy 1989), inherit a canonical form (Bastin and Gevers 1988, Marino 1990) or is linear and is realized in a canonical form (Kreisselmeier 1977). In this paper, we propose an adaptive observer algorithm for linearly parameterized systems. The observer does not require any special rank condition or canonical form. However, an output measurement condition is imposed. It is applicable to a special set of dynamic systems without any transformations. Therefore, the physical meaning of the states and parameters is 
preserved. Furthermore, since we use plant state-space representation, the proposed method is multi-input-multi-output (MIMO) in nature.

The remainder of this paper is organized as follows. In section 2, we show the problem definition. In section 3, the adaptive algorithms are derived and analyzed. Section 4 provides the simulation results for a hypothetical nonlinear system and a vehicle handling example. Finally, conclusions are given in section 5.

\section{Problem Formulation}

Consider a special class of MIMO nonlinear time invariant systems

$$
\left.\begin{array}{rl}
\dot{x} & =A x+\Gamma(y, u) \theta \\
y & =C x \\
A & =A_{n}+\Delta A
\end{array}\right\}
$$

where $x \in R^{n}$ denotes the state vector, $y \in R^{m}$ is the output vector, $C \in R^{m \times n}$ is the output matrix, $u \in R^{d}$ is the known input vector, $\theta \in R^{p}$ is the unknown parameter vector, $\Gamma(y, u) \in R^{n \times p}$ is a piecewise continuous nonlinear function, $\Delta A \in R^{n \times n}$ is the state matrix perturbation, and $A_{n} \in R^{n \times n}$ is the nominal state matrix. The following assumptions are made:

i) $\theta$ and $A$ are unknown but time-invariant, and their bounds are known.

ii) $\left(A_{n}, C\right)$ is an observable pair.

iii) The state vector $x$ is bounded.

The objective of the adaptive observer is to construct estimated $x(\hat{x}), \theta(\hat{\theta})$ and $A(\hat{A})$ such that $\hat{x} \rightarrow x, \hat{\theta} \rightarrow \theta$ and $\hat{A} \rightarrow A$ under zero exogenous disturbances. It should be noted that Eq.(1) has a special form. This special form is quite stringent for nonlinear systems. However, all linear systems can be put into the form of Eq.(1) (see second example of section 4.2). Therefore, the proposed methods can be applied to a reasonably large set of systems.

\section{Adaptive State and Parameter Estimation Algorithm}

The proposed adaptive state observer is:

$$
\dot{\hat{x}}=\left(A_{n}+\Delta \hat{A}\right) \hat{x}+\Gamma(y, u) \hat{\theta}+K(y-\hat{y})
$$

Let $E_{i}$ denotes a singleton column vector $\left[\begin{array}{llllll}0 & \cdots & 0 & 1(i t h) & 0 & \cdots\end{array}\right]^{T}$, we then have $\Delta \hat{A} \hat{x}=$ $\sum_{i=1}^{n} \sum_{j=1}^{n} E_{i} \hat{x}_{j}(t) \Delta \hat{A}_{i, j}$ and $\Delta A x=\sum_{i=1}^{n} \sum_{j=1}^{n} E_{i} x_{j}(t) \Delta A_{i, j}$, where $\Delta A_{i, j}$ is the $(i, j)$ th element of matrix $\Delta A$. The nonlinear uncertainty terms can be rewritten as $\Gamma(y, u) \hat{\theta}=\sum_{i=1}^{p} \Gamma_{i}(y, u) \hat{\theta}_{i}$ and $\Gamma(y, u) \theta=\sum_{i=1}^{p} \Gamma_{i}(y, u) \theta_{i}$, where $\Gamma_{i}$ is the $i$ th column of matrix $\Gamma$. Using these new representations, Eqs.(1) and (3) become 


$$
\begin{aligned}
& \dot{x}=A_{k} x+\sum_{i=1}^{n} \sum_{j=1}^{n} E_{i} x_{j}(t) \Delta A_{i, j}+\sum_{i=1}^{p} \Gamma_{i}(t) \theta_{i}+K y(t) \\
& \dot{\hat{x}}=A_{k} \hat{x}+\sum_{i=1}^{n} \sum_{j=1}^{n} E_{i} \hat{x}_{j}(t) \Delta \hat{A}_{i, j}+\sum_{i=1}^{p} \Gamma_{i}(t) \hat{\theta}_{i}+K y(t)
\end{aligned}
$$

where $A_{k}=A_{n}-K C$ is assumed to be Hurwitz by properly selected $\mathrm{K}$.

\section{Fact 3.1:}

If $\Delta \hat{A}$ and $\hat{\theta}$ are constant, there exist "augmented states" $\psi_{E_{i} \hat{x}_{j}}, \psi_{\Gamma_{i}}, \psi_{y}$ and $\psi_{E_{i} x_{j}}$ so that $\hat{x}(t)$ and $x(t)$ can be represented as $\hat{x}(t)=\Psi(t) \hat{\Theta}+\psi_{y}(t)+\exp \left(A_{k} t\right) \hat{x}(0)$, and

$$
\begin{gathered}
x(t)=\Psi \Theta+\psi_{y}(t)+\sum_{i=1}^{n} \sum_{j=1}^{n}\left(\psi_{E_{i} x_{j}}-\psi_{E_{i} \hat{x}_{j}}\right) \Delta A_{i, j}+\exp \left(A_{k} t\right) x(0) \text {. The augmented states are } \\
\dot{\psi}_{E_{i} \hat{x}_{j}}=A_{k} \psi_{E_{i} \hat{x}_{j}}+E_{i} \hat{x}_{j}(t) \\
\dot{\psi}_{\Gamma_{i}}=A_{k} \psi_{\Gamma_{i}}+\Gamma_{i}(t) \\
\dot{\psi}_{y}=A_{k} \psi_{y}+K y(t) \\
\dot{\psi}_{E_{i} x_{j}}=A_{k} \psi_{E_{i} x_{j}}+E_{i} x_{j}(t)
\end{gathered}
$$

which are grouped to define the matrices $\Psi=\left[\begin{array}{llllll}\psi_{E_{1} \hat{x}_{1}} & \cdots & \psi_{E_{n} \hat{x}_{n}} & \psi_{\Gamma_{1}} & \cdots & \psi_{\Gamma_{p}}\end{array}\right]$, $\hat{\Theta}=\left[\begin{array}{llllll}\Delta \hat{A}_{1,1} & \cdots & \Delta \hat{A}_{n, n} & \hat{\theta}_{1} & \cdots & \hat{\theta}_{p}\end{array}\right]^{T}$ and $\Theta=\left[\begin{array}{llllll}\Delta A_{1,1} & \cdots & \Delta A_{n, n} & \theta_{1} & \cdots & \theta_{p}\end{array}\right]^{T}$.

Proof: see Appendix.

Note that the "augmented states" are alternative representation of system dynamics, rather than an artificial implementation in the observer. From Fact 3.1, we have

$$
\begin{aligned}
& y=C\left(\Psi(t) \Theta+\psi_{y}(t)+\sum_{i=1}^{n} \sum_{j=1}^{n}\left(\psi_{E_{i} x_{j}}-\psi_{E_{i} \hat{x}_{j}}\right) \Delta A_{i, j}+\exp \left(A_{k} t\right) x(0)\right) \\
& \hat{y}=C\left(\Psi(t) \hat{\Theta}+\psi_{y}(t)+\exp \left(A_{k} t\right) \hat{x}(0)\right)
\end{aligned}
$$

Since $\left(A_{n}, C\right)$ is observable, the poles of $A_{k}$ can be arbitrarily selected. Therefore, the effect of initial conditions can be neglected. From Eqs.(10) and (11) we can clearly see that there is a disturbance term

$$
\mathrm{d}=\sum_{i=1}^{n} \sum_{j=1}^{n} C\left(\psi_{E_{i} x_{j}}-\psi_{E_{i} \hat{x}_{j}}\right) \Delta A_{i, j}
$$

which prevents the estimated outputs from converging to the true values. The effect of $\mathrm{d}$ can be limited by reducing $\psi_{E_{i} x_{j}}-\psi_{E_{i} \hat{x}_{j}}$. This is achieved by a properly designed $A_{k}$. The relationship between $\psi_{E_{i} x_{j}}-\psi_{E_{i} \hat{x}_{j}}$ and $A_{k}$ is shown in the following fact.

Fact 3.2: If (i) $A_{n}+\Delta \hat{A}-K C$ is Hurwitz, and (ii) $\Gamma(y, u) \in \mathcal{L}^{\infty}$, then we have $x(t)-\hat{x}(t) \in \mathcal{L}^{\infty}$ and $\lim _{t \rightarrow \infty}\left\|e_{\psi(i, j)}(t)\right\|_{2} \leq \frac{\mathcal{M}_{j}}{\sqrt{\lambda_{\text {min }}\left(A_{k}^{t} A_{k}\right)}}$ where $\left|x_{j}-\hat{x}_{j}\right| \leq \mathcal{M}_{j}, \quad e_{\psi(i, j)}(t)=\psi_{E_{i} x_{j}}-\psi_{E_{i} \hat{x}_{j}}$ and $\|\cdot\|_{2}$ denotes the vector 2-norm.

Proof: see Appendix. 
It should be noted that the assumption " $A_{n}+\Delta \hat{A}-K C$ is Hurwitz" in general is a nontrivial task and involves a careful search over the bounds of uncertainties to identify the solution of a simultaneous stabilization problem (SSP). The SSP can be solved by at least two approaches: either by solving a strong stabilizability problem (Vidyasagar 1985) or check whether the Kharitonov polynomials are Hurwitz (Kharitonov 1979). For the vehicle example to be shown in section 4.2 , however, the robust stabilization problem is trivial (see Eq.(18)).

\section{Proposition 3.3:}

If the assumptions of Fact 3.2 are satisfied, the least squares adaptive law under the cost function $J=\sum_{i=1}^{m} \int_{0}^{t} \mathrm{e}^{-\lambda(t-\tau)} e_{y i}(\tau)^{2} d \tau$ has the form $\dot{\hat{\Theta}}=\mathscr{P} \Psi_{c}^{T} e_{y}$ and $\dot{\mathcal{P}}=-\mathscr{P} \Psi_{c}^{T} \Psi_{c} \mathcal{P}+\lambda \mathscr{P}$ where $\Psi_{c}=C \Psi, e_{\Theta} \equiv \Theta-\hat{\Theta}, e_{y} \equiv y(t)-\hat{y}(t)$ and $\lambda>0$ is the forgetting factor.

Proof: See Appendix.

\section{Proposition 3.4:}

If (i) the assumptions of Fact 3.1 and Fact 3.2 are satisfied; and (ii) the adaptive laws shown in Proposition 3.3 are applied, then $\hat{\Theta}$ is bounded. Furthermore, if $\Psi_{c}$ is persistently exciting and $A_{k}$ is designed so that $e_{y}^{T} e_{y}>\mathrm{d}^{T} \mathrm{~d}$, then $x-\hat{x} \rightarrow 0$ and $\Theta-\hat{\Theta} \rightarrow 0$.

Proof: See Appendix.

\section{Simulation Results}

Two examples are presented below to verify the algorithms: a hypothetical nonlinear system and a vehicle handling example.

\subsection{A Hypothetical Nonlinear System}

The hypothetical nonlinear plant is assumed to have the form

$$
\begin{aligned}
& \frac{d}{d t}\left[\begin{array}{l}
x_{1} \\
x_{2}
\end{array}\right]=\left[\begin{array}{ll}
0 & 1 \\
a_{1} & a_{2}
\end{array}\right]\left[\begin{array}{l}
x_{1} \\
x_{2}
\end{array}\right]+\left[\begin{array}{l}
0 \\
b
\end{array}\right] u+\left[\begin{array}{l}
0 \\
c
\end{array}\right] \sin \left(x_{1}\right) \\
& y=x_{1}
\end{aligned}
$$

where $a_{1}, a_{2}, b$ and $c$ are unknown time-invariant parameters. The adaptive observer is then

$$
\frac{d}{d t}\left[\begin{array}{l}
\hat{x}_{1} \\
\hat{x}_{2}
\end{array}\right]=\left[\begin{array}{cc}
0 & 1 \\
a_{n 1} & a_{n 2}+\Delta \hat{a}_{2}
\end{array}\right]\left[\begin{array}{l}
\hat{x}_{1} \\
\hat{x}_{2}
\end{array}\right]+\left[\begin{array}{c}
0 \\
\Delta \hat{a}_{1}
\end{array}\right] y+\left[\begin{array}{l}
0 \\
\hat{b}
\end{array}\right] u+\left[\begin{array}{l}
0 \\
\hat{c}
\end{array}\right] \sin (y)+K(y-\hat{y})
$$

From the adaptive algorithm shown in Section 3, the unknown parameters and augmented states are $\hat{\Theta}=\left[\begin{array}{llll}\Delta \hat{a}_{2} & \Delta \hat{a}_{1} & \hat{b} & \hat{c}\end{array}\right]^{T}$ and $\Psi_{c}=C\left[\begin{array}{llll}\psi_{E_{2} y} & \psi_{E_{2} \hat{x}_{2}} & \psi_{E_{2} u} & \psi_{E_{2} \sin (y)}\end{array}\right]$. The adaptive laws $\dot{\hat{\Theta}}=\mathscr{P} \Psi_{c}^{T} e_{y}$ and $\dot{\mathcal{P}}=-\mathscr{P} \Psi_{c}^{T} \Psi_{c} \mathscr{P}+\lambda \mathcal{P}$ are applied, where $\mathcal{P}$ is a $4 \times 4$ matrix and $\lambda=0.15$. The parameters are assumed to be $a_{1}=-4, a_{2}=-5, b=4.5, c=-4$ and $a_{n 1}=-7, a_{n 2}=-$ $2, \hat{b}(0)=1.5$ and $\hat{c}(0)=-1$. The observer gain are assumed to be $K=\left[\begin{array}{ll}-5 & 5\end{array}\right]^{T}$. The initial values of $x$ and $\hat{x}$ are chosen to be $\hat{x}(0)=\left[\begin{array}{ll}0 & 2\end{array}\right]^{T}, x(0)=\left[\begin{array}{ll}0 & 0\end{array}\right]^{T}$, respectively. An input $u=1.1 \sin (0.3 t)+1.2 \sin (t)+\sin (1.5 t)$ is assumed to excite the plant. Simulation results illustrated in Figures 1 and 2 show that both states and parameters are accurately estimated. 


\subsection{Vehicle Handling Example}

A two degree-of-freedom vehicle lateral model (Figure 3, commonly known as the bicycle model) is studied in this example. The model lumps the front and rear wheels into axles, neglect the vertical, roll and pitch motions and assumed that the forward speed is constant. When lateral tire force is assumed to be proportional to the tire slip angle, this model becomes linear. The dynamics are described in the following state-space equation (Peng and Tomizuka 1990):

$$
\left[\begin{array}{c}
\dot{v}_{y} \\
\dot{r}
\end{array}\right]=\left[\begin{array}{cc}
-\frac{1}{m}\left(\frac{2 C_{f}+2 C_{r}}{v_{x}}\right) & \frac{1}{m}\left(-m v_{x}+\frac{-2 a C_{f}+2 b C_{r}}{v_{x}}\right) \\
\frac{1}{I_{z}}\left(\frac{-2 a C_{f}+2 b C_{r}}{v_{x}}\right) & \frac{1}{I_{z}}\left(\frac{-2 a^{2} C_{f}-2 b^{2} C_{r}}{v_{x}}\right)
\end{array}\right]\left[\begin{array}{c}
v_{y} \\
r
\end{array}\right]+\left[\begin{array}{c}
\frac{2 C_{f}}{m} \\
\frac{2 a C_{f}}{I_{z}}
\end{array}\right] u(15)
$$

where $v_{x}$ is the vehicle forward speed, $v_{y}$ is the vehicle lateral velocity, $r$ is the yaw rate, $m$ is the vehicle mass, $I_{z}$ is the yaw moment of inertia, $C_{f}$ and $C_{r}$ are the front and rear tire cornering stiffness. $u$ is the front wheel steering angle, and $a$ and $b$ are the distance from vehicle center of gravity to front and rear axles, respectively. Rewrite Eq.(15) into the linearly parameterized form, we have

$$
\begin{aligned}
& \dot{x}=A x+u \theta \\
& y=r
\end{aligned}
$$

where $x=\left[\begin{array}{c}v_{y} \\ r\end{array}\right], A \equiv\left[\begin{array}{ll}a_{1} & a_{3} \\ a_{2} & a_{4}\end{array}\right]$, and $\theta=\left[\begin{array}{cc}\frac{2 C_{f}}{m} & \frac{2 a C_{f}}{I_{z}}\end{array}\right]^{T}$. The adaptive observer is then

$$
\dot{\hat{x}}=\left(A_{n}+\Delta \hat{A}_{x}\right) \hat{x}+\Delta \hat{A}_{y} r+\left[\begin{array}{c}
-v_{x} \\
0
\end{array}\right] r+u \hat{\theta}+K(y-\hat{y})
$$

where $\quad \hat{y}=\hat{r}, \quad A_{n}=\left[\begin{array}{cc}-\left(\frac{2 C_{f n}+2 C_{r n}}{v_{x} m_{n}}\right) & \left(\frac{-2 a_{n} C_{f n}+2 b_{n} C_{r n}}{v_{x} m_{n}}\right) \\ \left(\frac{-2 a_{n} C_{f n}+2 b_{n} C_{r n}}{v_{x} I_{z n}}\right) & \left(\frac{-2 a_{n}^{2} C_{f}-2 b_{n}^{2} C_{r}}{v_{x} I_{z n}}\right)\end{array}\right], \quad \Delta \hat{A}_{x}=\left[\begin{array}{cc}\Delta \hat{a}_{1} & 0 \\ \Delta \hat{a}_{2} & 0\end{array}\right] \quad$ and $\Delta \hat{A}_{y}=\left[\begin{array}{ll}\Delta \hat{a}_{3} & \Delta \hat{a}_{4}\end{array}\right]^{T}$. The observer gain is $K=\frac{k}{v_{x}}$, where $k$ is a constant $2 \times 1$ matrix, i.e.,

$$
A_{n}-K C=\frac{1}{v_{x}}\left[\begin{array}{cc}
-\left(\frac{2 C_{f n}+2 C_{r n}}{m_{n}}\right) & \left(\frac{-2 a_{n} C_{f n}+2 b_{n} C_{r n}}{m_{n}}\right)-k_{1} \\
\left(\frac{-2 a_{n} C_{f n}+2 b_{n} C_{r n}}{I_{z n}}\right) & \left(\frac{-2 a_{n}^{2} C_{f}-2 b_{n}^{2} C_{r}}{I_{z n}}\right)-k_{2}
\end{array}\right]
$$

which is always stable when $k_{1}$ and $k_{2}$ are properly selected (i.e., the robust stabilization problem is easily solved). The steering input is assumed to have a pseudo-sinusoidal form as shown in Fig. 4. Two cases are studied.

\section{Case (1):}


This case presents the nominal condition with only the driver in the car, and the vehicle is driven on a dry road surface. The parameter values are $C_{f n}=71000 \mathrm{~N} / \mathrm{rad}$, $C_{r n}=68000 \mathrm{~N} / \mathrm{rad}, a_{n}=1.32 \mathrm{~m}, b_{n}=1.58 \mathrm{~m}, m_{n}=1900 \mathrm{~kg}, I_{z n}=3100 \mathrm{~kg}-\mathrm{m}^{2}$ and $v_{x}=15 \mathrm{~m} / \mathrm{sec}$. It can be seen from Figures 5 and 6 that the vehicle states and parameters are accurately estimated.

\section{Case (2):}

For this case we assume that the tire cornering stiffness is reduced and the vehicle load is increased. The perturbed parameter values are $C_{f}=51000 \mathrm{~N} / \mathrm{rad}, C_{r}=50000 \mathrm{~N} / \mathrm{rad}$, $a=1.49 \mathrm{~m}, b=1.41 \mathrm{~m}, m=2230 \mathrm{~kg}, I_{z}=3844 \mathrm{~kg} \mathrm{~m} \mathrm{~m}^{2}$ and $v_{x}=15 \mathrm{~m} / \mathrm{sec}$. The observer gain $\left[\begin{array}{ll}k_{1} & k_{2}\end{array}\right]^{T}=\left[\begin{array}{ll}-10 & 10\end{array}\right]^{T}$, and the forgetting factor $\lambda=0.15$. Besides the parameter variations described above, we also assume that measurement noises exist. The steering angle is assumed to be contaminated by a white noise (standard deviation $=0.1$ degree), and the yaw rate noise is also white (standard deviation=0.005 rad $/ \mathrm{sec}$ ). The results are shown in Figures 7 and 8. Again, good estimation results are obtained.

\section{Conclusions}

An output feedback adaptive observer was proposed in this paper. The main idea behind this method is that the system output can be represented as a linear function of unknown parameters and augmented states. A least squares method is applied to estimate parameter values, which are then used to improve state estimation. The estimated states and parameters were found to converge to the true values when the input signals are sufficiently rich and the state observer gain satisfies certain conditions. Two simulation examples were studied--a hypothetical nonlinear system and a linear vehicle handling problem. In both cases, states and parameters were accurately estimated.

\section{Acknowledgment}

This work is supported by DOT under the contract DTFH61-93-X-00017 through the ITS Research Center of Excellence at the University of Michigan.

\section{References}

Bastin, G., and Gevers, M. R., 1988, "Stable Adaptive Observers for Nonlinear Time-Varying Systems," IEEE Trans. on Automatic Control, vol. 33, no. 7, pp. 650-658.

Dugard, L., and Goodwin, G.C., 1985, "Global Convergence of Landau's "Output Error with Adjustable Compensator" Adaptive Algorithm," IEEE Trans. on Automatic Control, vol. 30, no. 6, pp. 593-595.

Kharitonov, V.L., 1979, "Asymptotic Stability of an Equilibrium Position of a Family of Systems of Linear Differential Equations," Differential Equations Vol.14, pp.1483-1485.

Kreisselmeier, G., 1977, “Adaptive Observers with Exponential Rate of Convergence,” IEEE Trans. on Automatic Control, vol. 22, no. 1, pp. 2-8.

Kudva, P., and Narendra, K.S. 1973, "Synthesis of an Adaptive Observer Using Lyapunov's Direct Method,” Int. J. Control, vol. 18, pp.1201-1210.

Luders, G. and Narendra, K. S., 1973, “An Adaptive Observer and Identifier for a Linear System,” IEEE Trans. on Automatic Control, vol. 18, no. 5, pp. 496-499. 
ASME Journal of Dynamic Systems, Measurement and Control, Vol. 120 n4 Dec 1998, pp.524-528

Luenberger, D. G., 1971, “An Introduction to Observers," IEEE Trans. on Automatic Control, vol. AC-16, no. 6, pp. 596-602.

Marino, R., 1990, “Adaptive Observers for Single Output Nonlinear Systems,” IEEE Trans. on Automatic Control, vol. 35, no. 9, pp. 1054-1058.

Marino, R., and Tomei, P., 1995, "Adaptive Observers with Arbitrary Exponential Rate of Convergence for Nonlinear Systems," IEEE Trans. on Automatic Control, vol. 40, no. 7, pp. 13001304.

Narendra , K. S. and Annaswamy, A. M., 1989, Stable Adaptive Systems, Prentice-Hall, Englewood Cliffs, New Jersey.

Peng, H. and Tomizuka, M., 1993, "Preview Control for Vehicle Lateral Guidance in Highway Automation," ASME J. of Dynamic Systems, Measurement and Control, Vol.115, No.4, pp.679-686.

Popov, V. M., 1973, Hyperstability of Control Systems, Springer-Verlag, New York.

Vidyasagar, M., 1985, Control System Synthesis, The MIT Press, Cambridge, MA. 


\section{Proof of Fact 3.1:}

\section{Appendix}

Assuming that $\Delta \hat{A}$ and $\hat{\theta}$ are fixed, the Laplace transformation of Eq.(3) is

$$
\left(s I-A_{k}\right) \hat{x}(s)=\sum_{i=1}^{n} \sum_{j=1}^{n} E_{i} \hat{x}_{j}(s) \Delta \hat{A}_{i, j}+\sum_{i=1}^{p} \Gamma_{i}(s) \hat{\theta}_{i}+K y(s)+\hat{x}(0)
$$

Let $E_{i} \hat{x}_{j}(s)=\left(s I-A_{k}\right) \psi_{E_{i} \hat{x}_{j}}(s), \Gamma_{i}(s)=\left(s I-A_{k}\right) \psi_{\Gamma_{i}}(s)$ and $K y(s)=\left(s I-A_{k}\right) \psi_{y}(s)$, Eq.(A.1) can be rewritten as

$$
\begin{aligned}
\hat{x}(t) & =\sum_{i=1}^{n} \sum_{j=1}^{n} \psi_{E_{i} \hat{x}_{j}}(t) \Delta \hat{A}_{i, j}+\sum_{i=1}^{p} \psi_{\Gamma_{i}}(t) \hat{\theta}_{i}+\psi_{y}(t)+\exp \left(A_{k} t\right) \hat{x}(0) \\
& =\Psi(t) \hat{\Theta}+\psi_{y}(t)+\exp \left(A_{k} t\right) \hat{x}(0)
\end{aligned}
$$

where $\Psi=\left[\begin{array}{llllll}\psi_{E_{1} \hat{x}_{1}} & \cdots & \psi_{E_{n} \hat{x}_{n}} & \psi_{\Gamma_{1}} & \cdots & \psi_{\Gamma_{p}}\end{array}\right]$ and $\hat{\Theta}=\left[\begin{array}{llllll}\Delta \hat{A}_{1,1} & \cdots & \Delta \hat{A}_{n, n} & \hat{\theta}_{1} & \cdots & \hat{\theta}_{p}\end{array}\right]^{T}$. The plant dynamics (Eq.(1)) can then be written as

$$
\dot{x}(t)=A_{k} x(t)+\sum_{i=1}^{n} \sum_{j=1}^{n} E_{i} x_{j}(t) \Delta A_{i, j}+\sum_{i=1}^{p} \Gamma_{i}(t) \theta_{i}+K y(t)
$$

Or, in the s-domain:

$$
\left(s I-A_{k}\right) x(s)=\sum_{i=1}^{n} \sum_{j=1}^{n} E_{i} x_{j}(s) \Delta A_{i, j}+\sum_{i=1}^{p} \Gamma_{i}(s) \theta_{i}+K y(s)+x(0)
$$

From Eqs.(7),(8),(9), and (A.4), we have

$$
\begin{aligned}
x(t) & =\sum_{i=1}^{n} \sum_{j=1}^{n} \psi_{E_{i} x_{j}}(t) \Delta A_{i, j}+\sum_{i=1}^{p} \psi_{\Gamma_{i}}(t) \theta_{i}+\psi_{y}(t)+\exp \left(A_{k} t\right) x(0) \\
& =\Psi \Theta+\psi_{y}(t)+\sum_{i=1}^{n} \sum_{j=1}^{n}\left(\psi_{E_{i} x_{j}}-\psi_{E_{i} \hat{x}_{j}}\right) \Delta A_{i, j}+\exp \left(A_{k} t\right) x(0)
\end{aligned}
$$

\section{Proof of Fact 3.2:}

Subtract Eq.(3) from Eq.(1), we have

$$
\begin{aligned}
& \dot{e}_{x}=\left(A_{n}-K C\right) e_{x}+\Delta A x(t)-\Delta \hat{A} \hat{x}(t)+\Gamma(y, u)(\theta-\hat{\theta}) \\
& =\left(A_{n}+\Delta \hat{A}-K C\right) e_{x}+[\Delta A-\Delta \hat{A}] x(t)+\Gamma(y, u)(\theta-\hat{\theta})
\end{aligned}
$$

where $e_{x} \equiv x(t)-\hat{x}(t)$. If $A_{n}+\Delta \hat{A}-K C$ is Hurwitz and $\hat{\theta}, \Gamma(y, u)$ and $x(t)$ are bounded, $e_{x}$ is bounded. Assuming that $\|\exp (\tilde{A} t)\|_{2} \leq \exp \left(-\lambda_{m} t\right) \quad$ and $\|(\Delta A-\Delta \hat{A}) x(t)+\Gamma(y, u)(\theta-\hat{\theta})\|_{2} \leq M$, it is easy to show that $\exists \tau, \forall t>\tau,\left\|e_{x}(t)\right\|_{2} \leq \frac{M}{\lambda_{m}}$, which implies $\left|x_{j}(t)-\hat{x}_{j}(t)\right| \leq M_{j}, \forall t>\tau$. Subtract Eq.(6) from Eq.(9), we have

$$
\dot{e}_{\psi(i, j)}(t)=A_{k} e_{\psi(i, j)}(t)+E_{i}\left(x_{j}-\hat{x}_{j}\right)
$$


where $e_{\psi(i, j)}(t)=\psi_{E_{i} x_{j}}-\psi_{E_{i} \hat{x}_{j}}$ The solution of Eq.(A.7) is

$$
e_{\psi(i, j)}(t)=\exp \left(A_{k} t\right) e_{\psi(i, j)}(0)+\int_{0}^{\tau} \exp \left[A_{k}(t-\tau)\right] E_{i}\left[x_{j}(\tau)-\hat{x}_{j}(\tau)\right] d \tau
$$

The Euclidean norm of which is bounded by

$$
\begin{aligned}
\left\|e_{\psi(i, j)}(t)\right\|_{2} & \leq\left\|\exp \left(A_{k} t\right) e_{\psi(i, j)}(0)\right\|_{2}+\left\|\int_{0}^{\tau} \exp \left[A_{k}(t-\tau)\right] d \tau\right\|_{2}\left\|E_{i}\right\|_{2}\left\|\mathcal{M}_{j}\right\|_{2} \\
& \leq\left\|\exp \left(A_{k} t\right) e_{\psi(i, j)}(0)\right\|_{2}+\frac{\left\|\exp \left(A_{k} t\right)-I\right\|_{2}\left\|E_{i}\right\|_{2}\left\|\mathcal{M}_{j}\right\|_{2}}{\sqrt{\lambda_{\min }\left(A_{k}^{T} A_{k}\right)}}
\end{aligned}
$$

Since $A_{k}$ is Hurwitz and $\left\|E_{i}\right\|_{2}=1$, after the transient dies out, we have

$$
\lim _{t \rightarrow \infty}\left\|e_{\psi(i, j)}(t)\right\|_{2} \leq \frac{\mathcal{M}_{j}}{\sqrt{\lambda_{\min }\left(A_{k}^{T} A_{k}\right)}}
$$

\section{Proof of Proposition 3.3:}

The cost function is assumed to have the form

$$
J=\sum_{i=1}^{m} \int_{0}^{t} \mathrm{e}^{-\lambda(t-\tau)} e_{y i}(\tau)^{2} d \tau
$$

where $\lambda>0$ is a forgetting factor. To minimize this cost function, we need to satisfy

$$
\frac{\partial J}{\partial \hat{\Theta}}=2 \sum_{i=1}^{m} \int_{0}^{t} \mathrm{e}^{-\lambda(t-\tau)} \psi_{c i}^{T}(\tau)\left[\psi_{c i}(\tau) \hat{\Theta}+\psi_{c y i}-y_{i}(\tau)\right] d \tau=0
$$

where $\left.\mid \begin{array}{ccc}\leftarrow & \psi_{c 1} & \rightarrow \\ \vdots & \\ \leftarrow & \psi_{c m} & \rightarrow\end{array}\right]=C \Psi$ and $\psi_{c y i}$ is the $i$ th element of $C \psi_{y}$. From Eq.(A.12), the least squares estimate is

$$
\hat{\Theta}=\left[\sum_{i=1}^{m} \int_{0}^{t} \mathrm{e}^{-\lambda(t-\tau)} \psi_{c i}^{T}(\tau) \psi_{c i}(\tau) d \tau\right]^{-1}\left[\sum_{i=1}^{m} \int_{0}^{t} \mathrm{e}^{-\lambda(t-\tau)} \psi_{c i}^{T}(\tau)\left[y_{i}(\tau)-\psi_{c y i}(\tau)\right] d \tau\right](\mathrm{A}
$$

To avoid matrix inversion in real-time, define

$$
\mathscr{P}=\left[\sum_{i=1}^{m} \int_{0}^{t} \mathrm{e}^{-\lambda(t-\tau)} \psi_{c i}^{T}(\tau) \psi_{c i}(\tau) d \tau\right]^{-1}
$$

It is then easy to show that $\dot{P}=-\mathcal{P} \sum_{i=1}^{m} \psi_{c i}^{T} \psi_{c i} \mathcal{P}+\lambda \mathcal{P}=-\mathcal{P} \Psi_{c}^{T} \Psi_{c} \mathcal{P}+\lambda \mathcal{P}$. Eq. (A.13) can then be written as

$$
\hat{\Theta}=\mathscr{P}\left[\sum_{i=1}^{m} \int_{0}^{t} \mathrm{e}^{-\lambda(t-\tau)} \psi_{c i}^{T}(\tau)\left[y_{i}(\tau)-\psi_{c y i}(\tau)\right] d \tau\right]
$$


The time derivative of which is then

$$
\dot{\hat{\Theta}}=-\mathscr{P} \sum_{i=1}^{m} \psi_{c i}^{T}(t)\left[\psi_{c i}(t) \hat{\Theta}+\psi_{c y i}(t)-y_{i}(t)\right]
$$

Substitute $\hat{y}_{i}(t)=\psi_{c i}(t) \hat{\Theta}+\psi_{c y i}(t)$ into Eq.(A.16), the least square parameter update law is then $\dot{\hat{\Theta}}=\mathscr{P} \sum_{i=1}^{m} \psi_{c i}^{T} e_{y i}=\mathscr{P} \Psi_{c}^{T} e_{y}$.

\section{Proof of Proposition 3.4:}

Choose the Lyapunov candidate $V=\frac{1}{2} e_{\Theta}^{T} \mathcal{P}^{-1} e_{\Theta}$, we have

$$
\dot{V}=-e_{\Theta}^{T} \Psi_{c}^{T} e_{y}+\frac{1}{2} e_{\Theta}^{T} \Psi_{c}^{T} \Psi_{c} e_{\Theta}-\frac{1}{2} \lambda e_{\Theta}^{T} \mathcal{P}^{-1} e_{\Theta}=-\lambda V-e_{\Theta}^{T} \Psi_{c}^{T} e_{y}+\frac{1}{2} e_{\Theta}^{T} \Psi_{c}^{T} \Psi_{c} e_{\Theta}
$$

Subtract Eq.(11) form Eq.(10) and neglect the term related to the initial condition, we obtain

$$
\Psi_{c} e_{\Theta}=e_{y}-\mathrm{d}
$$

where $e_{y} \equiv y-\hat{y}$ and $e_{\Theta} \equiv \Theta-\hat{\Theta}$. From Eqs.(A.17) and (A.18), we have

$$
\dot{V}=-\lambda V-\frac{1}{2} e_{y}^{T} e_{y}+\frac{1}{2} d^{T} d
$$

Therefore, $\dot{V}<0$ iff $-2 \lambda V-e_{y}^{T} e_{y}<-d^{T} d$. A sufficient condition for this inequality to hold true is $-e_{y}^{T} e_{y}<-d^{T} d$. When $\dot{V}<0$ we have $e_{\Theta}(t) \rightarrow 0, d(t) \rightarrow 0$ and $e_{x}(t) \rightarrow 0$ as $\mathrm{t} \rightarrow \infty$. 
ASME Journal of Dynamic Systems, Measurement and Control, Vol. 120 n4 Dec 1998, pp.524-528

\section{List of Figures}

Fig. 1. State Estimation Results of Example 1

Fig. 2 Parameter Estimation Results of Example 1

Fig. 3 The vehicle bicycle model

Fig. 4 Front Wheel Steering Angle

Fig . 5 State estimation results for Example 2, Case 1 (Nominal case)

Fig . 6 Parameter estimation results for Example 2, Case 1 (Nominal case)

Fig . 7 State estimation results for Example 2, Case 2 (Perturbed case)

Fig . 8 Parameter estimation results for Example 2, Case 2 (Perturbed case) 


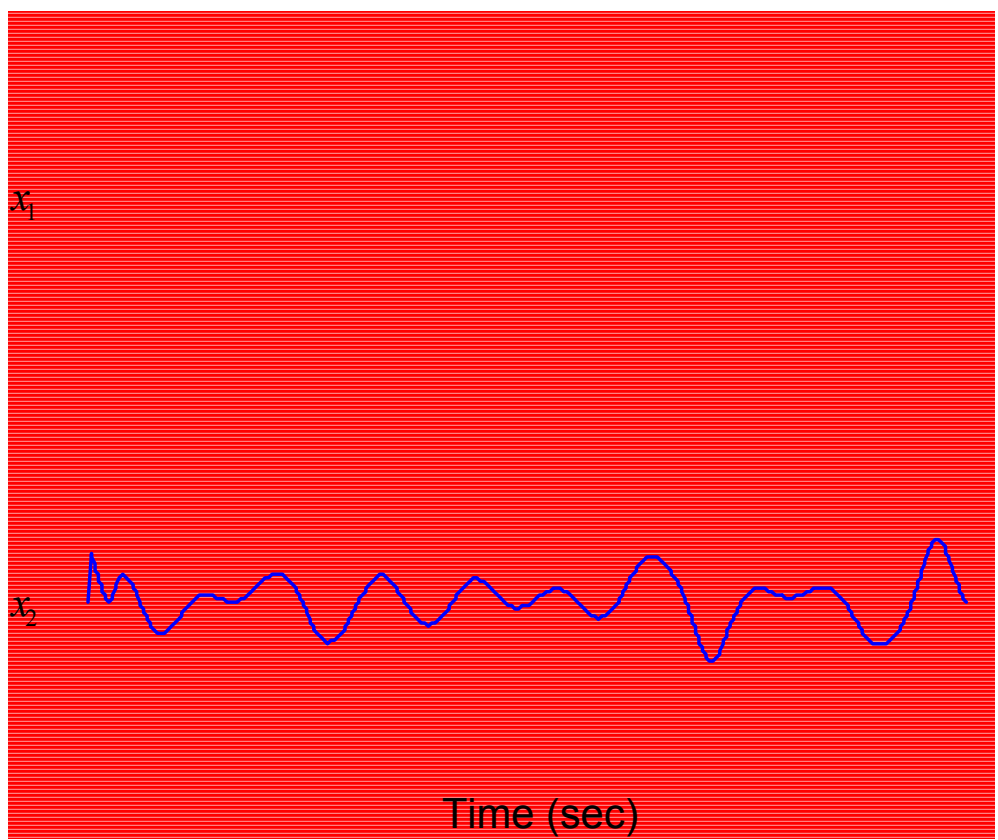

Fig. 1. State Estimation Results of Example 1
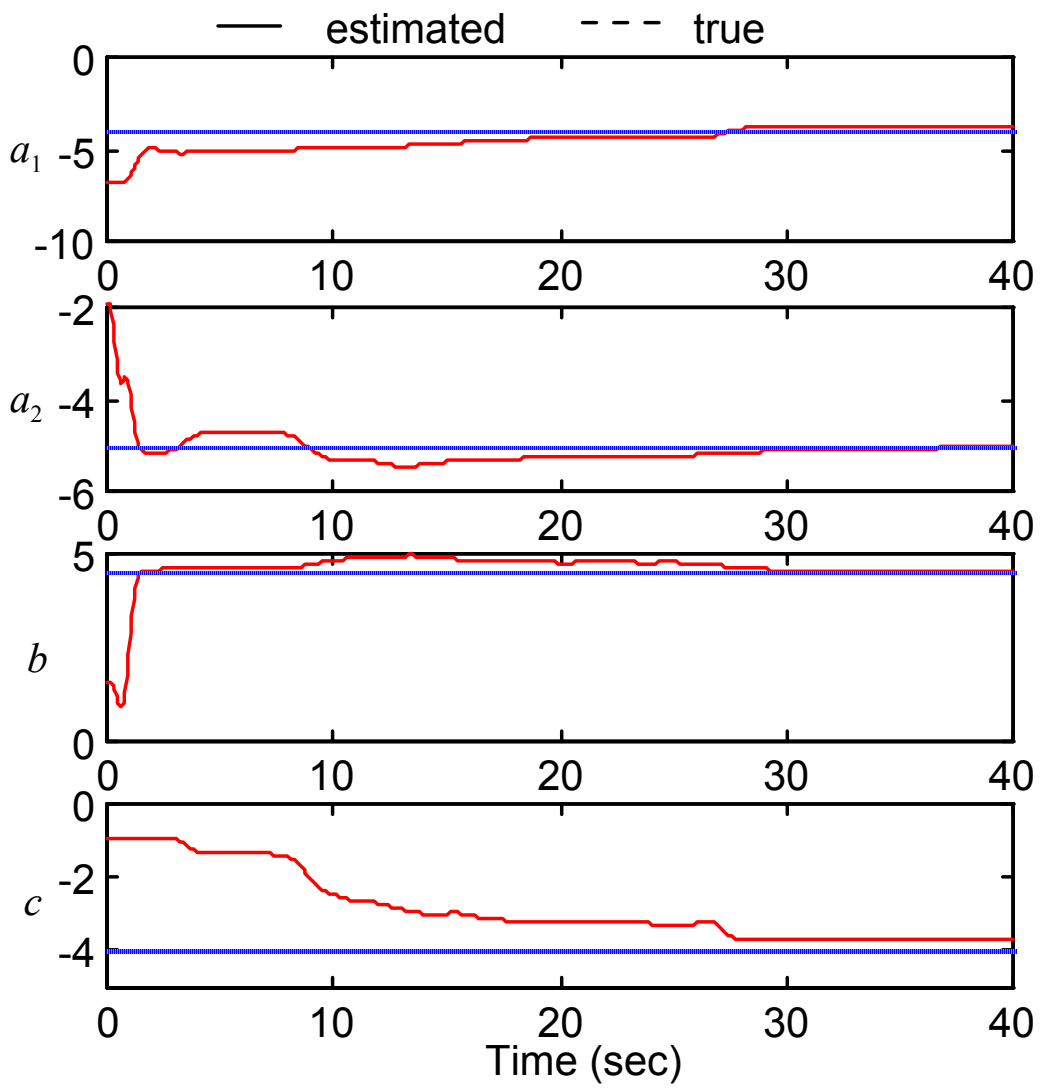

Fig. 2 Parameter Estimation Results of Example 1 


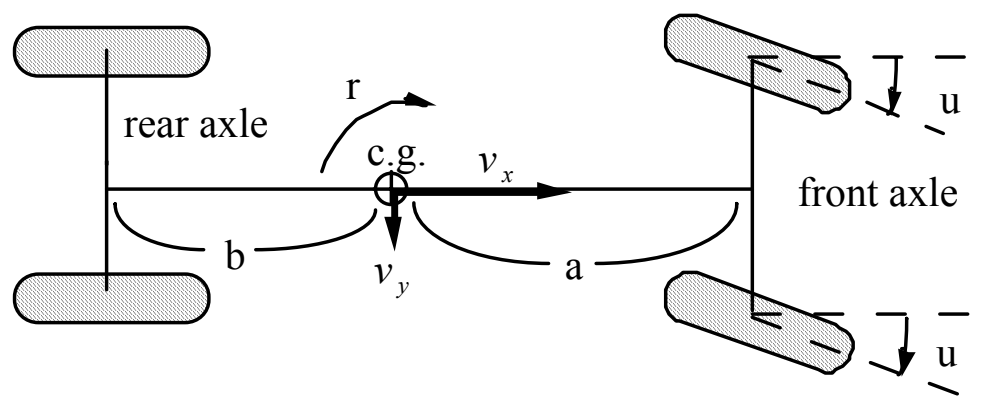

Fig. 3 The vehicle bicycle model

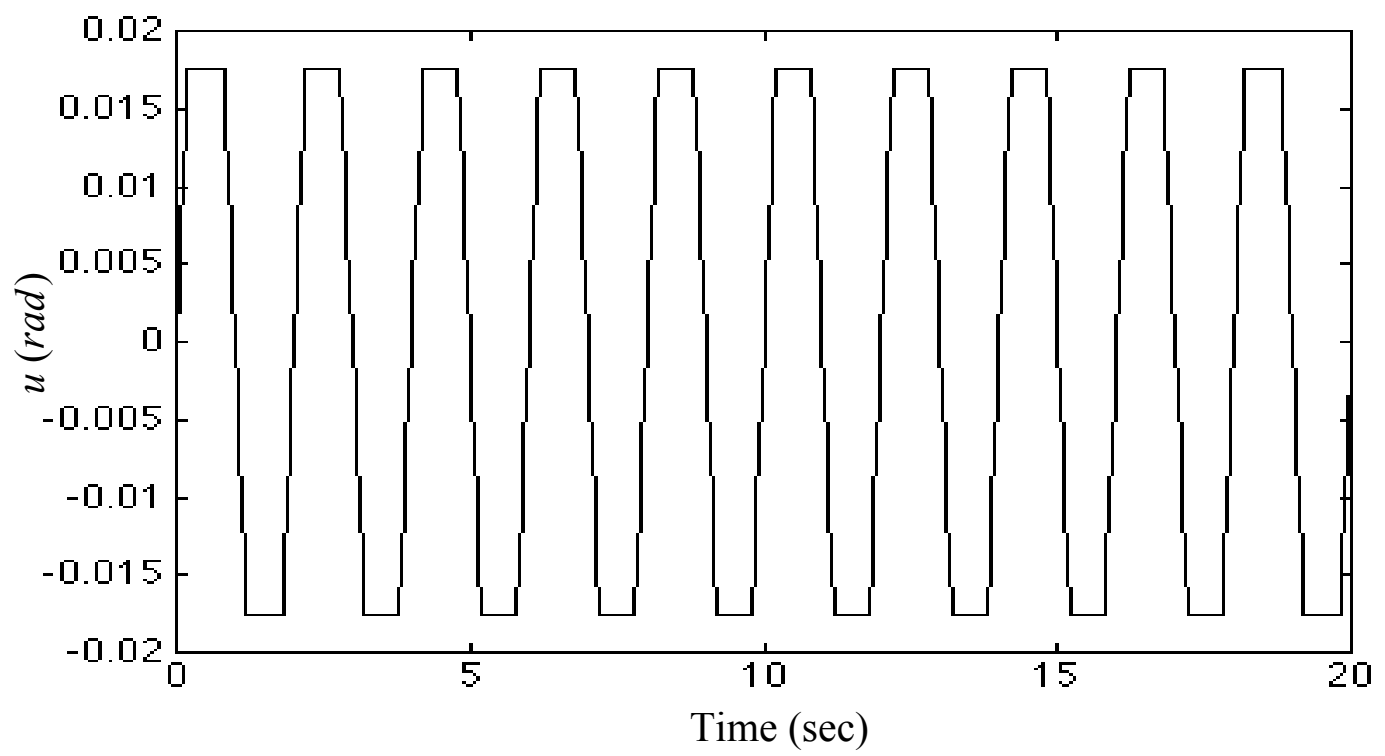

Fig. 4 Front Wheel Steering Angle 

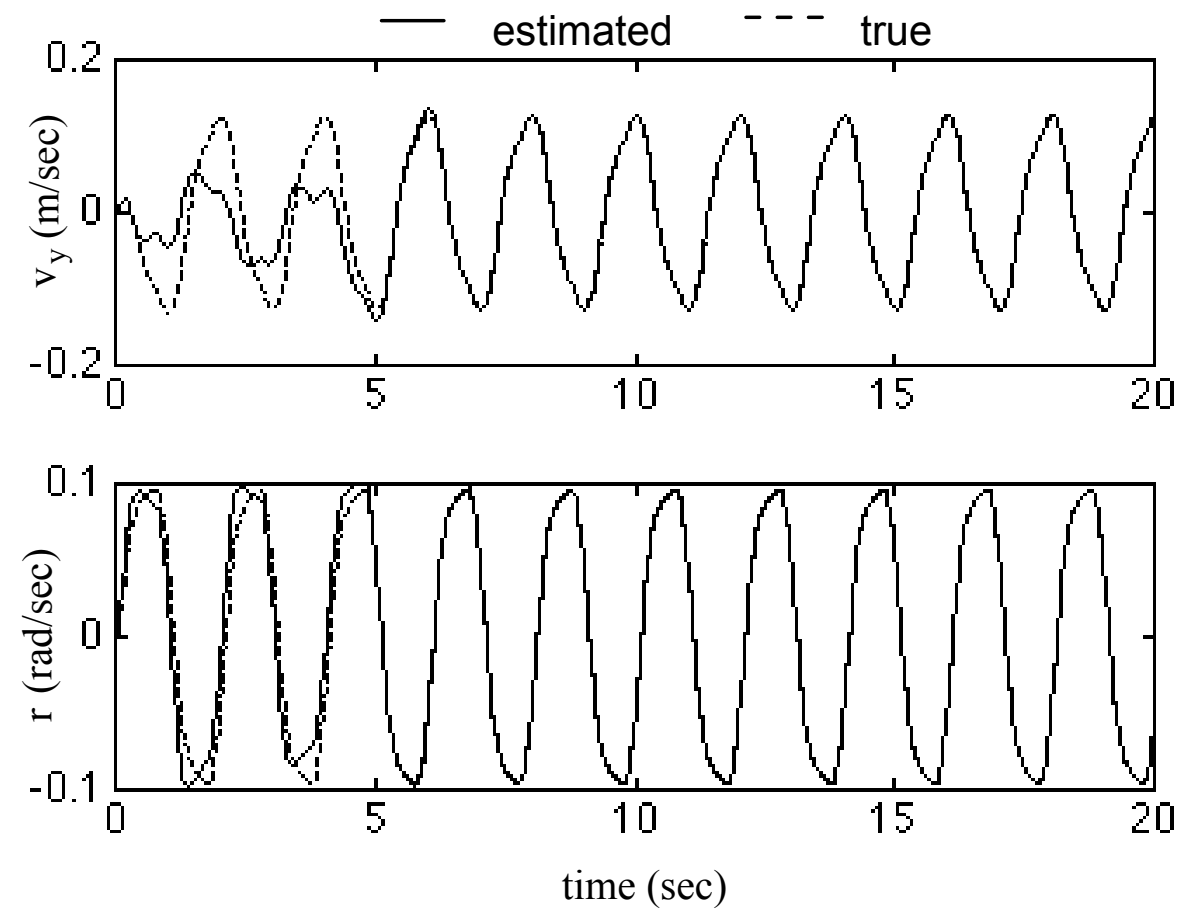

Fig . 5 State estimation results for Example 2, Case 1 (Nominal case)
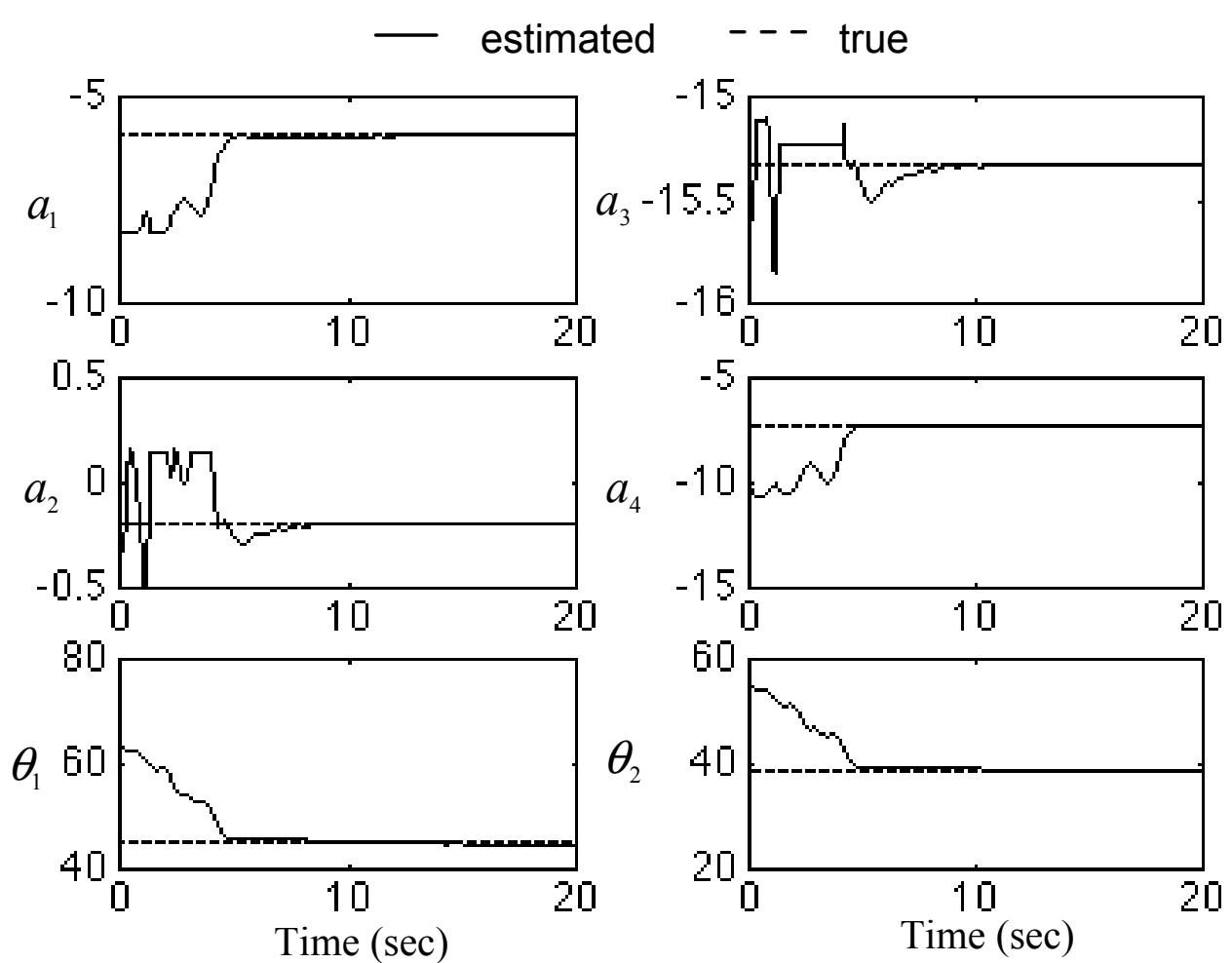

Fig . 6 Parameter estimation results for Example 2, Case 1 (Nominal case) 

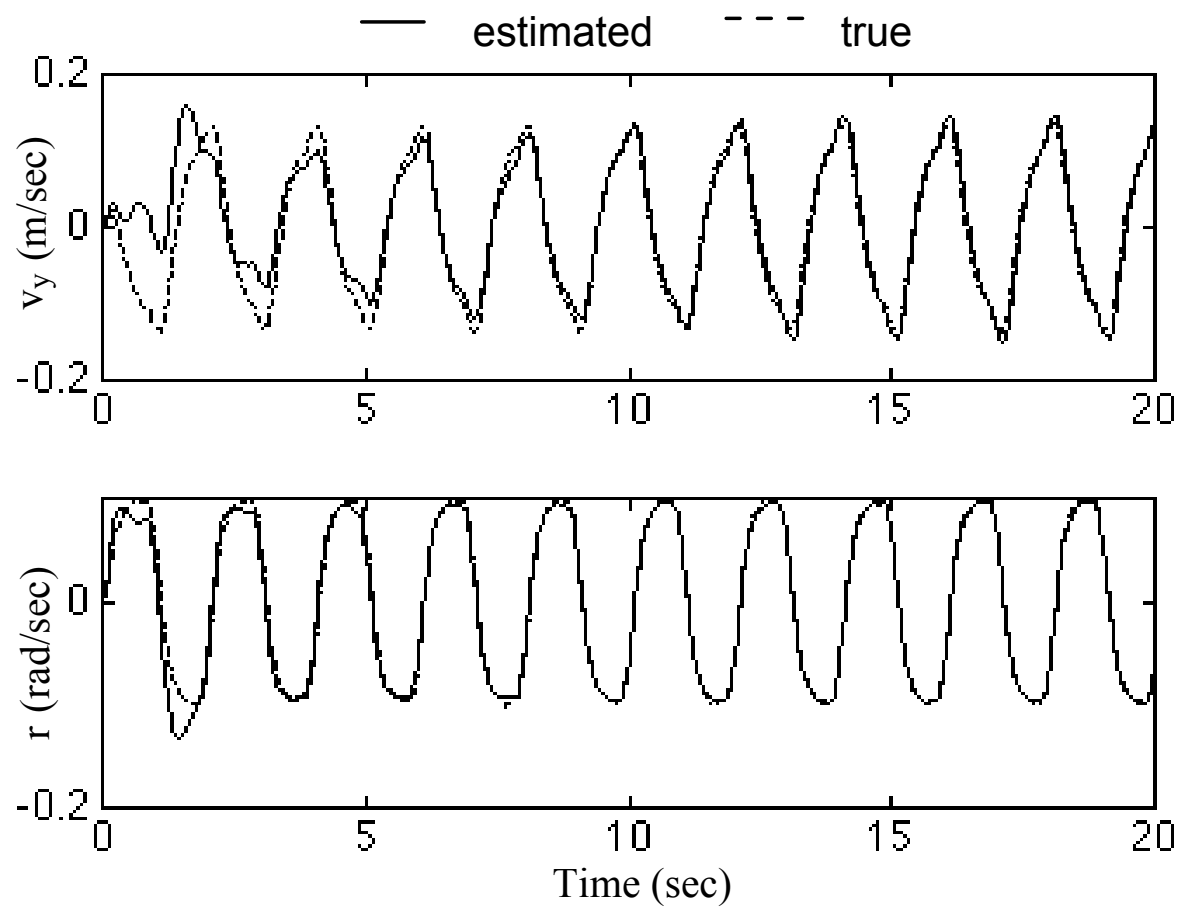

Fig . 7 State estimation results for Example 2, Case 2 (Perturbed case)

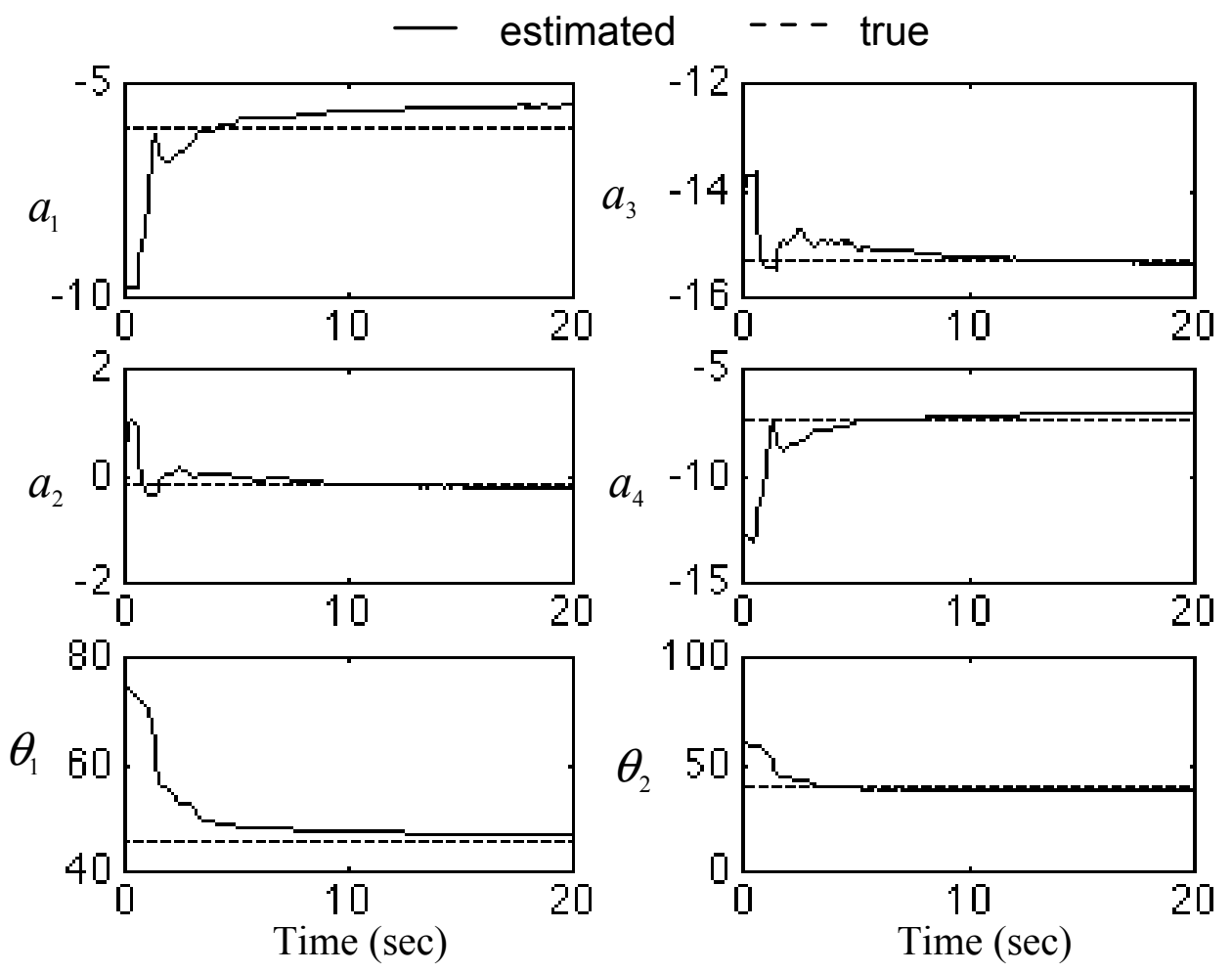

Fig . 8 Parameter estimation results for Example 2, Case 2 (Perturbed case) 